\title{
Predicting Charging Load of Pure Electric Buses Based on Multi-distribution Statistics
}

\author{
Qu TianYi, a \\ ${ }^{1}$ School of Management, XuZhou University of Technology, Xuzhou, Jiangsu, China 221008 \\ ajdbh2001@163.com
}

\begin{abstract}
Keywords: Pure electric buses; Combination of fast and slow speed; Charging mode; Monte Carlo load forecasting
\end{abstract}

\begin{abstract}
Compared with family cars and taxis, buses have more regular features such as driving time, space, and distance. In a large number of statistics on the running and charging rules of pure electric buses, and on the basis of fully considering the power consumption and opening time of the hot and cold air-conditioning of pure electric buses, hybrid charging mode , the combination of the normal nighttime charging for a pure electric bus and the fast charging during work, is obtained. Monte Carlo method is used to predict the charging load of pure electric vehicles, which provides a reference for the grid to formulate relevant countermeasures to reduce the impact of electric vehicle access on the grid.
\end{abstract}

\section{Introduction}

To a certain extent, electric vehicles can alleviate the problems of energy shortages and environmental crises [1] and have been promoted by governments of various countries. With the continuous development of the follow-up electric vehicles, large-scale electric vehicles will have a certain impact on the power grid [2-4], when accessing to it. If we know the charging rules of electric vehicles ahead of time, facilitate will be preparation in advance. At present, the research methods of the charging law of electric vehicles are mainly divided into three categories: 1) Comprehensive analysis methods such as literature [5]. This method estimates the development scale of electric vehicles based on certain development trends, derived from big data. It is simple and convenient, while takes no consideration on the specific impact of the development of electric vehicles; 2) specific analysis methods such as the literature [6], a series of assumptions on the common four types of electric vehicles are limited, in this range given the general rules of charging, this method is more limited 3) Probability analysis method Such as literature [7], based on the law of electric vehicle travel, the main influencing factors of electric vehicle charging load are summed up and fitted to obtain regular probability distribution parameters. The use of this method, compared to the other two methods results, is more popular and closer to the actual, but the inductive and fitting of various influencing factors in the previous period requires a lot of data, the authenticity and real-time nature of the data, which is difficult to study. So this method has more room for improvement.

\section{Factors Affecting the Charging Load of Pure Electric Buses}

Through the analysis of the data of a large number of pure electric buses, combined with the driving and refueling characteristics of existed fuel vehicles, it can be seen that the main factors affecting the charging load of pure electric buses are the initial charging time, the charging power of electric vehicles and charging piles, Day mileage, climate, etc.

Electric buses have fixed working hours and driving routes relative to family cars and taxis, so it is relatively stable in time and space. Charging sites are generally located at the bus departure station, and the initial-charging time is the arrived time.

During the work period of the bus, there is no long time for regular charging, so it is necessary to fill or charge the battery in a short time to the amount of battery that can support the bus to go back 
and forth. However, after the buses have been shut down from work, there is a longer parking time. In order to reduce the impact on the power grid, regular charging is proposed.

Air conditioner is the second largest energy-consuming device on electric vehicles. The operating modes of air-conditioning in different seasons are different. Since the consumption of air-conditioning energy is affected by many factors, such as the number of people on the vehicle, the size of the vehicle, and the setting of the air-conditioning temperature, these factors influence each other, so it is difficult to find specific rules of consumption of air-conditioning power caused by the environment temperature.

\section{The Establishment of a Charging Load Model}

(1) Assuming that under the premise that the power grid does not participate in the regulation of bus charging, we set the conventional charging power $P_{1}$ and high-power fast charging power $P_{2}$, the maximum mileage of the bus on a daily basis $D_{m}$, the battery capacity of the bus $C_{N}$, the charging efficiency of the charging pile $K$, and the energy consumption of the air conditioning $K_{\alpha}$.

(2) Initializing the number of simulations $N$ and record the number of simulations starting from $\mathrm{n}=1$, and satisfying $n \leq N$;

(3) Initializing the number of electric buses (M) in an area, $m=1$, which means starting from the first bus and satisfying $m \leq M$;

(4) According to formula 2, extracting the number of kilometers on the public car mileage D;

(5) Combining formulas 4, 5, 6, and 7 to calculate the charge duration $T_{c}$.

(6) According to the formula 1 to extract the bus starting charging time $T_{s}$, indicating a charging time period, in $[0,144]$ between, when the initial charging time plus the charging time exceeds 144 , the charging end time shall be subtracted by 144 , that is $T_{e}=T_{e}-144$, time is the next day;

(7) For the calculation of power, the calculation formula for the charging power of the m-th bus is as follows:

$$
P_{m}=\sum_{i=T_{s}}^{T_{e}} P(m, i)+P(m, \alpha)
$$

Among them, $T_{s}$ and $T_{e}$ are obtained by rounding off, $P(m, \alpha)$ indicates the air-conditioning energy consumption of the $m$-th bus.

(8) Determine whether all bus charging power are superimposed, when $m=M$, it indicates that superposition is complete, then go to step (9) . Otherwise, the calculation of all charging loads has not been completed,yet $m<M$, and $m$ need to update ,then go to step (4).

(9) Determine whether to complete the set number of simulations, when $n=M$, it indicates that the number of simulations reached, then go to step (10). Otherwise, simulation has not been finished,yet $n<M$, and nneed to update , $n=n+1$ then go to step (3).

(10) Judging whether or not to converge. Calculate the accuracy of the charging load by the variance coefficient. The formula for calculating the variance coefficient is as follows:

$$
\varpi=\frac{\beta}{\alpha \sqrt{N}}
$$

Setting Monte Carlo simulation times to 5000 , $\mathrm{n}=5000$. Calculate the step size in 10 minutes and divide the day into 144 time periods. 


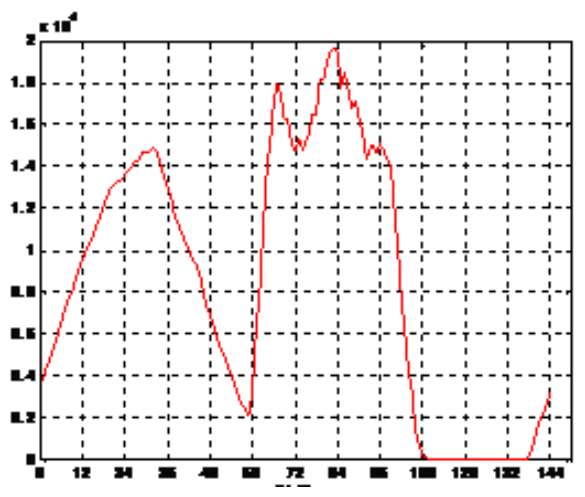

Fig. 1 Charging load curve of buses

When the electric buses are scheduled to perform normal nighttime charging, it not only share the pressure of the daytime charging stations, but also help the filling of the power grid. If the power grid participates in charge regulation, the charging strategy is formulated to avoid the daily peak load time as much as possible, which will greatly reduce the impact of electric bus access to the grid.

\section{Conclusions}

Since the bus has a certain regularity in time and space distribution ,compared with the family car and taxi, the charging location and the charging start time can be roughly determined, so the orderly charging of the bus can be carried out smoothly. When V2G[15] technology is used, buses can be managed centrally. When the grid is at peak load, the buses are discharged to the grid. When the grid is in a valley load, a large number of electric buses are charged and the electric energy is stored, attaining peaking load shift. At this stage, electric buses are not yet universally available. The number of electric buses is limited, and the amount of energy that can be stored is relatively small compared to the daily load of the power grid. Even if V2G technology is implemented, the effect will not be obvious.

\section{References}

[1] xiaolin su ,yanjuanzhang ,zhongwu, xiaoxiayan, Prediction of charging load of large-scale electric vehicles and its influence on power grid, J. Modern Electric Power.2018,35(01):45-54.

[2] litingtian, mingxiazhang, huanlingwang, Evaluation and Solution of Electric Vehicle Impact on Power Grid,J.Proceeding of the CSEE. 2012,32(31):43-49+217

[3] zechunhu, yonghua song, zhiweixu, zhuoweiluo, qiqiaozhan, Influence and Utilization of Electric Vehicles' Access to Power Grid, J.Proceeding of the CSEE.2012,32(04):1-10+25.

[4] huling li, xiaominbai,Influence of Electric Vehicle Charging on Distribution Network and Countermeasures, J. Automation of Electric Power System.2011,35(17):38-43.

[5] zhuoweiluo, zechunhu, yonghua song, xia yang, qizhanqiao, Electric vehicle charging load calculation method, J. Automation of Electric Power System.2011,35(14):36-42.

[6] Kristien C N, Edwin H, Johan D. The impact of charging plug-in hybrid electric vehicles on a residential distribution grid, J. IEEE Trans on Power Systems, 2010, 25(1): 371-380.

[7] xin gong, taolin, binghuasu, The Impact of Plug-in Hybrid Electric Vehicle Charging on Distribution Network, J.Power System Technology, 2012,36(11):30-35.

[8] defuwang, shubingzhang, Time-space Distribution Prediction of Electric Vehicle Charging Load and Analysis of Influence on Power Network Load, J. Journal Electric Power. 2017,32(06):483-489.

[9] senwang,yaohanfeng, wenxiongdeng, gang zhou, penggei sheng, Electric vehicle charging load prediction considering the distribution of time and space characteristics, J.Zhejiang Electric Power.2016,35(12):15-20. 